\title{
The jet and X-ray corona of Cygnus X-1
}

\author{
Julien Malzac* \\ CESR (CNRS/Universite de Toulouse) \\ E-mail: malzacecesr.fr
}

Cygnus X-1 is probably the best studied black hole in the universe. The wealth of accurate data may be used to constrain the models of black hole accretion and ejection. I show that the current estimates for the jet power of Cygnus X-1 set a lower limit on the jet bulk velocity. These estimates also suggest that the X-ray emission does not arise in the jet, as was proposed by several authors. However the jet could contribute at higher energy and be responsible for the emission reported at a $\mathrm{TeV}$ energies by MAGIC. Regarding the X-ray emitting corona, it is well known that the temperature and optical depth of the Comptonising electrons can be measured using spectroscopy in the $1 \mathrm{keV}-1 \mathrm{MeV}$ energy band. I will show that other physical parameters of the corona, such as the strength of magnetic field, or the temperature of the ions can be constrained as well. In the prototypical source Cynus X-1, the results appear to challenge current accretion models.

The Extreme sky: Sampling the Universe above $10 \mathrm{keV}$

October 13-17 2009

Otranto (Lecce) Italy

${ }^{*}$ Speaker. 


\section{Introduction}

Black hole binaries are observed in two main X-ray spectral states, namely the Hard State (HS) and the Soft State (SS; see Done, Gierlinski and Kubota 2007). The hard X-ray emission in both spectral states is well represented by Comptonisation by an hybrid thermal/non-thermal electron distribution. In the HS the temperature and optical depth of the thermal electrons are higher, and the slope of the non-thermal tail seem steeper than in the SS. Consequently, the X-ray emission is dominated by thermal Comptonisation in the HS and by non-thermal Comptonisation in the SS. The HS is known to be associated with the presence of a compact radio jet which is not observed in the SS. Here we focus on the prototypical black hole source Cyg X-1. In section 2, we present a relatively simple coupled kinetic-radiation model that allows us to understand the origin of the very different spectral shapes observed in the two spectral state as well as the spectral evolution during state transitions (see e.g. Del Santo et al. 2008). A thorough investigation of the model and its discussion in the context of the observations can be found in Malzac \& Belmont (2009), the present paper summarises our main results. Then in section 3, I summarise the arguments developed by Malzac, Belmont and Fabian (2009) showing that the present estimates of the jet power of Cygnus $\mathrm{X}-1$ imply that the jet has a relativistic velocity, that the accretion proceeds efficiently in the hard state and that the X-ray emission is unlikely to be produced in the jet.

\section{Magnetic field and ion temperature in the corona}

The code of Belmont et al. (2008) solves the kinetic equations for photons, electrons and positrons in the one-zone approximation. It accounts for Compton scattering (using the full KleinNishima cross section), $e^{+}-e^{-}$pair production and annihilation, Coulomb interactions (electronelectron and electron-proton), synchrotron emission and absorption and $e-p$ bremsstrahlung. Radiative transfer is dealt using a usual escape probability formalism. We model the Comptonising region as a sphere with radius $R$ of fully ionised proton-electron magnetised plasma in steady state. The Thomson optical depth of the sphere is $\tau_{\mathrm{T}}=\tau_{\mathrm{i}}+\tau_{\mathrm{s}}$, where $\tau_{\mathrm{i}}=n_{i} \sigma_{\mathrm{T}} R$ is the optical depth of ionisation electrons (associated with protons of density $n_{i}$ ) and $\tau_{s}=2 n_{\mathrm{e}^{+}} \sigma_{\mathrm{T}} R$ is the optical depth of electrons and positrons due to pair production $\left(n_{\mathrm{e}^{+}}\right.$is the positron number density). $\sigma_{\mathrm{T}}$ is the Thomson cross section. The radiated power is quantified through the usual compactness parameter $l=\frac{L \sigma_{\mathrm{T}}}{R m_{\mathrm{e}} c^{3}}$, where $L$ is the luminosity of the Comptonising cloud, $m_{\mathrm{e}}$ the electron rest mass and $c$ the speed of light. We consider three possible channels for the energy injection in our coupled electron-photon system: (i) Non-thermal electron acceleration with a compactness $l_{\text {nth }}$. We model the acceleration process by assuming electrons are continuously injected with a power-law distribution of index $\Gamma_{\text {inj. }}$. (ii) Coulomb heating with a compactness $l_{\mathrm{c}}$. Electrons are supposed to interact by Coulomb collisions with a distribution of thermal ions: $l_{\mathrm{c}}>0$ if the ions of the plasma have a larger temperature than the electrons. (iii) External soft radiation coming from the geometrically thin accretion disc and entering the corona with a compactness $l_{\mathrm{s}}$. Since, in this model, all the injected power ends up into radiation we have: $l=l_{\text {nth }}+l_{\mathrm{c}}+l_{\mathrm{s}}$ in steady state. In addtition the magnetic field $B$ is parametrized through the usual magnetic compactness: $l_{B}=\frac{\sigma_{\mathrm{T}}}{m_{\mathrm{e}} c^{2}} R \frac{B^{2}}{8 \pi}$. We find that a pure non-thermal injection model (i.e. assuming $l_{\mathrm{c}}=0$ ) provides a good description of the high energy spectra of Cygnus X-1 in both spectral states (see Fig 1). According to our models, the 


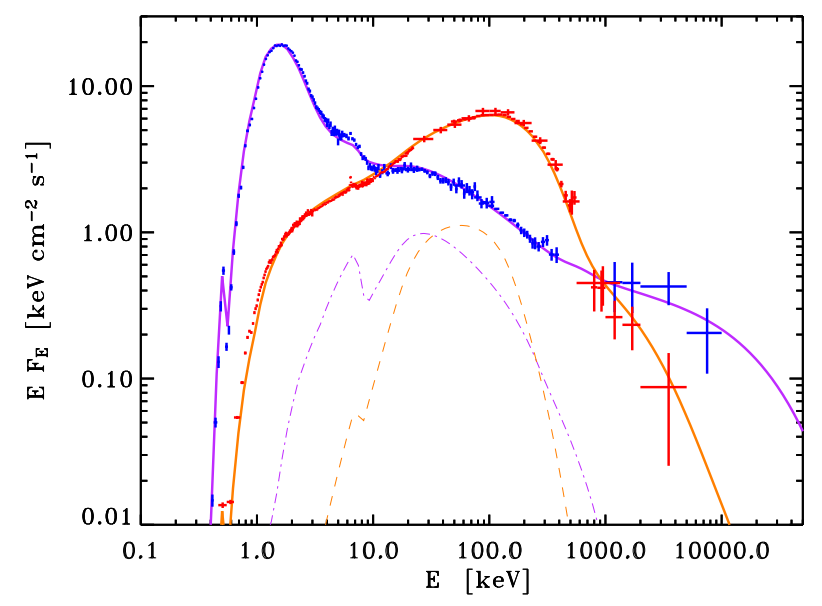

Figure 1: A comparison of the average CGRO spectra for the SS (blue) and HS (red) of Cygnus X-1 (data from McConnell et al., 2002), with models involving only injection of non-thermal particles as sole heating mechanism. At low energy the CGRO data are complemented by BeppoSAX. The thick purple line shows our absorbed SS model while the orange line shows the HS model of the same table. Reflection components were added to both spectra and are shown by the thin dot-dashed and dashed curves for the SS model and the HS model respectively.

non-thermal compactness of the corona is comparable $\left(l_{\text {nth }} \simeq 5\right)$ in both spectral states. As expected most of the differences between HS and SS are due to a change in the soft photon compactnes $l_{\mathrm{s}}$ that we assumed to be 0 and about $3 l_{\text {nth }}$ in the HS and SS respectively. We find the magnetic field must be relatively low in the HS (while it is not very well constrained in the SS). In fact if we compare the magnetic to radiation energy density in both models we find the $U_{B} / U_{\mathrm{R}} \simeq 3$ in the SS while in the HS, $U_{B} / U_{\mathrm{R}} \simeq 0.3$. In any case, the magnetic field inferred from our model in the HS is probably an upper limit on the actual magnetic field in the source. The presence of external soft photons (neglected in this fit) would imply a lower $B$ to keep the coronal temperature high. The fact that this maximum magnetic field appears significantly below equipartition with radiation suggests that the emission of the corona is not powered by the magnetic field, as assumed in most accretion disc corona models (e.g. di Matteo, Celotti \& Fabian 1997; Merloni \& Fabian 2001). Alternatively, models with heating by hot protons (i.e. $l_{\mathrm{c}}>0$ ) also provide a very good description of the spectra of Cygnus X-1. However even in these models some level of non-thermal acceleration is required in order to reproduce the non-thermal MeV tails. In our 'best fit' models about $25 \%$ of the heating power is provided in the form of non-thermal acceleration in the HS while this fraction rises to about $2 / 3$ in the SS. We infer ion temperature of about $50 \mathrm{MeV}$ in the SS versus only $1.3 \mathrm{MeV}$ in the HS. We note that in the HS the proton temperature appears significantly lower than what predicted by standard 2-temperature accretion flow solutions (the temperature of the hot protons in typical ADAF models is in the range $10-100 \mathrm{MeV}$ ).

\section{Constraints from the jet power}

Deep radio observations of the field of Cyg X-1 resulted in the discovery of a shell-like nebula which is aligned with the resolved radio jet (Gallo et al. 2005). This large-scale (5 pc in diameter) 
structure appears to be inflated by the inner radio jet. Gallo et al. (2005) estimate that in order to sustain the observed emission of the shell, the jet of Cyg X-1 has to carry a kinetic power that is comparable to the bolometric X-ray luminosity of the binary system. Russell et al. (2007) refined this estimate and found that the total kinetic power of the double sided jet is $L_{\mathrm{J}}=(0.9-3)$ $\times 10^{37} \mathrm{erg} \mathrm{s}^{-1}$. If we adopt $L_{h}=2 \times 10^{37} \mathrm{erg} \mathrm{s}^{-1}$ as the typical X-ray luminosity in the HS then $j=L_{\mathrm{J}} / L_{\mathrm{h}}$ is in the range 0.45-1.5. Malzac, Belmont \& Fabian (2009) show that this estimate of the jet power sets some constraints on the physics of the accretion and ejection in Cyg X-1 that we sumarise below.

\subsection{The accretion efficiency and jet velocity}

In the SS there is overwhelming evidence that accretion proceeds through a geometrically thin optically thick accretion disc. Therefore the accretion is efficient. Assuming that there is no jet in the SS, the efficiency can be defined as:

$$
\eta_{\mathrm{s}}=L_{\mathrm{s}} / \dot{M}_{\mathrm{s}} c^{2}
$$

where $L_{\mathrm{S}}$ is the source luminosity in the SS, $\dot{M}_{\mathrm{S}}$ the mass accretion rate. According to the theory of general relativity $\eta_{\mathrm{s}}$ is in the range $0.06-0.4$ depending on the spin of the black hole. In the HS, the accretion probably does not proceeds through a standard thin disc and the efficiency is essentially unknown. Depending on the nature of the accretion flow, it could be close to that of the SS or much smaller (as e.g. in an advection dominated accretion flow). If we take into account the presence of the energetically important jet, the efficiency in the HS can be formaly writen as:

$$
\eta_{\mathrm{h}}=\frac{L_{\mathrm{J}}+L_{\mathrm{h}}}{\left(1-f_{\mathrm{j}}\right) \dot{M}_{\mathrm{h}} c^{2}}
$$

where $f_{\mathrm{j}}$ represents the fraction of the accreting material which is ejected and cannot be used to release energy. Observationally we know that $L_{\mathrm{j}} \simeq L_{\mathrm{h}}$ and that $L_{\mathrm{s}} / L_{\mathrm{h}}<4$. Moreover in Cygnus X-1 the spectral transition is believed to be triggered by an increase in the accretion rate and therefore $\dot{M}_{\mathrm{s}}>\dot{M}_{\mathrm{h}}$. Combining this with equations (3.1) and (3.2), it follows that $\eta_{\mathrm{h}}>\eta_{\mathrm{s}} / 2$. This shows that accretion is quite efficient in the HS and cannot be strongly advection dominated.

The jet kinetic power can be written as:

$$
L_{\mathrm{J}}=f_{\mathrm{j}} \dot{M}_{\mathrm{h}}\left(\gamma_{\infty}-1\right) c^{2},
$$

combining this with equations (3.2) and (3.1) we get an estimate of the terminal bulk Lorentz factor of the jet:

$$
\gamma_{\infty}=1+\frac{j \eta_{\mathrm{s}}}{\lambda(1+j) \frac{\eta_{\mathrm{s}}}{\eta_{\mathrm{h}}}}
$$

where $j=L_{\mathrm{j}} / L_{\mathrm{h}} \simeq 1$ and $\lambda=\frac{L_{\mathrm{s}}}{L_{\mathrm{h}}} \frac{\dot{M}_{\mathrm{h}}}{\dot{M}_{\mathrm{h}}}<4$ For typical parameters (e.g. $\lambda \simeq 3$ and $\eta_{\mathrm{s}} \simeq \eta_{\mathrm{h}} \simeq 0.1$ ), this gives estimates of the terminal jet velocity in the range $0.3 c-0.8 c$. Such mildly relativistic velocities are in agreement with independent estimates based on radio observations (see Gleissner et al. 2004; Ibragimov et al. 2007). An absolute lower limit on the jet velocity $\beta_{\infty}=v_{\infty} / c>0.1$ is obtained in the extreme case $\eta_{\mathrm{h}}=1, \eta_{\mathrm{s}}=0.06, \lambda=4$ and $j=0.45$. The jet velocity is therefore at least mildly relativistic. 


\subsection{Does the $\mathrm{X}$-ray emission originate in the jet ?}

Several authors have suggested that the X-ray may actually be produced by the jet (e.g. Markoff et al. 2001; Georganopoulos, Aharonian \& Kirk 2002; Giannos et al. 2004; Kylafis et al. 2008). The jet energetics shows however that this is unlikely. Indeed, mass flux is conserved along the jet, and therefore the rate at which mass is ejected can be written as:

$$
\dot{M}_{\mathrm{J}}=\frac{L_{\mathrm{J}}}{\left(\gamma_{\infty}-1\right) c^{2}}=\pi r(z)^{2} n(z) \beta(z) \gamma(z) m_{\mathrm{p}} c<6.6 \times 10^{18} \quad \mathrm{~g} \mathrm{~s}^{-1}
$$

where $r, n, \beta$ and $\gamma$ represent the jet section, density, bulk velocity and Lorentz factor at a given height $z$ above the black hole. The upper limit of $6.6 \times 10^{18} \mathrm{~g} \mathrm{~s}^{-1}$ comes from the constraints $\beta_{\infty}>0.1$ and $L_{\mathrm{J}}<3 \times 10^{37} \mathrm{erg} \mathrm{s}^{-1}$. Spectral fits with Comptonisation models of the HS spectra of Cynus X-1 require a Thomson optical depth $\tau_{\mathrm{T}}$ in the range 1-3. Now let us assume that this Comptonised emission is produced somewhere in the jet at some height $z_{0}$. This gives the additional constraint:

$$
\tau_{T} \simeq n\left(z_{0}\right) \sigma_{T} r\left(z_{0}\right)>1
$$

where $\sigma_{\mathrm{T}}$ is the Thomson cross section. Combining this with the constraints from equation (3.5), we find that for any reasonable jet section (i.e. $>10 R_{G}$ ) the velocity of the $\mathrm{X}$-ray emiting region must be non relativistic, $\beta\left(z_{0}\right)<0.1$. In fact this upper limit is very conservative and for reasonable parameters we actually expect the velocity to be much lower than $0.1 \mathrm{c}$. The part of the jet producing the X-rays should be very slow and most of the jet bulk velocity would have to be acquired at larger distances from the black hole. However, most X-ray jet models require or assume both $\tau_{T}>1$ and initial velocity of the X-ray emitting region that is larger than $0.1 c$. As long as the estimates of Russell et al. (2007) are correct, these models can be ruled out. This conclusion however does not apply to the jet model of Markoff et al. (2001, 2005 herafter M05). Indeed, in this model the X-ray emission is produced through synchrotron self-Compton emission of very energetic electrons of temperature of a few $\mathrm{MeV}$ and optical depth $\tau_{\mathrm{T}} \sim 10^{-3}-10^{-2}$. The low Thomson depth makes it energetically possible to have a mildly relativistic initial jet velocity. We note however that such a combination of small size, very low optical depth and large temperature is physically impossible. Indeed in Cyg X-1, the large luminosity and small emitting region make the compactness larger than unity and electron positron production can be significant. For a compactness of order of a few (like in Cyg X-1), the optical depth must be at least 10 times larger than in this jet model (see discussion in Malzac et al. 2009). The parameters of the model of M05 are therefore inconsistent with the constraints from pair equilibrium. Finally we note that although the jet is unlikely to contribute significantly to the hard X-ray emission of Cygnus X-1, it may nevertheless be at the origin of the gamma-ray emission that was detected by the MAGIC telescope (Albert et al. 2007; Malzac et al. 2008; Zdziarski et al. 2009).

\section{Discussion}

In both spectral states of black hole binaries the coronal emission can be powered by a similar non-thermal acceleration mechanism. In the HS the synchrotron and $e$-e Coulomb boilers 
redistribute the energy of the non-thermal particles to form and keep a quasi-thermal electron distribution at a relatively high temperature, so that most of the luminosity is released through quasithermal comptonisation. In the SS, the soft photon flux from the accretion disc becomes very strong and cools down the electrons, reducing the thermal Compton emissivity. This change in the soft photon flux could be caused either because the inner radius of the truncated disc moves inward into the central hot accretion flow, or, in the framework of accretion disc corona models, because the disc temperature increases dramatically. Then most of the emission is produced by disc photons up-scattered by the non-thermal cooling electrons. Our comparison of simulations with the high energy spectra of Cygnus X-1 in the HS allowed us to set upper limits on the magnetic field and the proton temperature. Our results indicates that in the HS the magnetic field is below equipatition with radiation (unlike what is assumed in most accretion disc corona models). The proton temperature is found to be significantly lower than predicted by standard 2-temperature accretion flow models $\left(k T_{\mathrm{i}}<2 \mathrm{MeV}\right)$. We also note that such accretion flows are usually radiatively inneficient while the jet energetics suggests efficient accretion in the HS. The present estimates of the jet power also suggest that the jet is Thomson thin with a mildly relativistic velocity, which does not make it a favoured location for the production of the observed X-ray emission.

\section{References}

[1] Albert J., et al., 2007, ApJ, 665, L51

[2] Belmont, R., Malzac, J., \& Marcowith, A. 2008, A\&A, 491, 617

[3] Del Santo, M., et al. 2008, MNRAS, 390, 227

[4] Di Matteo T., Celotti A., Fabian A. C., 1999, MNRAS, 304, 809

[5] Done, C., Gierliński, M., \& Kubota, A. 2007, A\&A Rev., 15, 1

[6] Georganopoulos M., Aharonian F. A., Kirk J. G., 2002, A\&A, 388, L25

[7] Giannios D., Kylafis N. D., Psaltis D., 2004, A\&A, 425, 163

[8] Gierliński et al. 1999, MNRAS, 309, 496

[9] Gleissner T., et al., 2004, A\&A, 425, 1061

[10] Gallo E., et al. 2005, Natur, 436, 819

[11] Ibragimov A., Zdziarski A. A., Poutanen J., 2007, MNRAS, 381, 723

[12] Kylafis N. D., et al. 2008, A\&A, 489, 481

[13] Malzac J., et al. 2008, A\&A, 492, 527

[14] Malzac, J., \& Belmont, R. 2009, MNRAS, 392, 570

[15] Malzac J., Belmont R., Fabian A. C., 2009, MNRAS, 400, 1512

[16] Markoff S., Falcke H., Fender R., 2001, A\&A, 372, L25

[17] Markoff S., Nowak M. A., Wilms J., 2005, ApJ, 635, 1203

[18] McConnell M. L., et al., 2002, ApJ, 572, 984

[19] Merloni A., Fabian A. C., 2001, MNRAS, 321, 549

[20] Russell D. M., Fender R. P., Gallo E., Kaiser C. R., 2007, MNRAS, 376, 1341

[21] Zdziarski A. A., Malzac J., Bednarek W., 2009, MNRAS, 394, L41 